\title{
Adaptive Algorithms for Diagnosing Large-Scale Failures in Computer Networks
}

\author{
Srikar Tati *, Bong Jun $\mathrm{Ko}^{\dagger}$, Guohong Cao*, Ananthram Swami ${ }^{\ddagger}$, and Thomas La Porta * \\ *Network and Security Research Center, Pennsylvania State University,Email: $\{$ tati,gcao,tlp\}@cse.psu.edu \\ ${ }^{\dagger} I B M$ TJ Watson, Email: bongjun_ko@us.ibm.com; ${ }^{\ddagger}$ Army Research Laboratory, Email: aswami@arl.army.mil
}

\begin{abstract}
In this paper, we propose an algorithm to efficiently diagnose large-scale clustered failures. The algorithm, Cluster-MAX-COVERAGE (CMC), is based on greedy approach. We address the challenge of determining faults with incomplete symptoms. CMC makes novel use of both positive and negative symptoms to output a hypothesis list with a low number of false negatives and false positives quickly. CMC requires reports from about half as many nodes as other existing algorithms to determine failures with $100 \%$ accuracy. Moreover, CMC accomplishes this gain significantly faster (sometimes by two orders of magnitude) than an algorithm that matches its accuracy. Furthermore, we propose an adaptive algorithm called Adaptive-MAX-COVERAGE (AMC) that performs efficiently during both kinds of failures, i.e., independent and clustered. During a series of failues that include both independent and clustered, $\mathrm{AMC}$ results in a reduced number of false negatives and false positives.
\end{abstract}

Keywords-Fault diagnosis; Large-scale failures; Incomplete information; Clustered failures

Category: Regular paper with approximately 2350 words

I declare that the material has been cleared through the affiliations of the author(s).

\section{INTRODUCTION}

Critical steps in achieving high network reliability are detecting, diagnosing, and localizing faulty network elements [1] (e.g., network nodes, links) after failures. Conventional fault detection and diagnosis techniques [2], [3] deal with failures that are sporadic and independent in nature, i.e., caused by individual component failures (e.g., broken links, adapter failure, routing software malfunction, etc.). In this paper, we focus on large-scale failures in networks.

Large-scale failures are possible due to events such as intentional attacks and natural disasters. During natural disasters like Hurricanes Katrina and Irene [4], [5], earthquakes in Japan and Taiwan [6], [7], and electricity blackouts [5], there are reports that large portions of the Internet are damaged. In addition, intentional attacks (e.g., 9/11 attack [8], [9]) may damage a significant portion of a network, and create massive disruptions. We note that the type of outages caused by large-scale failures differ greatly from those caused by typical equipment faults [2], [3], [10]. Large-scale outages tend to create faults at multiple components that are geographically close to each other. We call these failures clustered failures.

There is considerable interest in large-scale failures in the current literature [11], [12], but not much work focuses on diagnosing these clustered failures. Due to discrepancy in failure patterns, the performance of fault diagnosis techniques that are focused on independent failures [3], [10] degrades when applied to clustered failures. A fault diagnosis algorithm called netCSI [13] is proposed to localize largescale failures in networks. It is shown that by considering the failure patterns of large-scale outages, this algorithm can achieve higher accuracy than existing algorithms developed for independent failures [10]. However, the drawback of netCSI is that the run-time complexity of the algorithm increases exponentially with the increase in size of networks since it is a combinatorial approach.

In this paper, we propose a new algorithm called ClusterMAX-COVERAGE (CMC) that diagnoses large-scale clustered failures. To identify the faulty network elements (i.e., network nodes, routers, and links) CMC utilizes a knowledge base of possible network paths and end-to-end symptom information. The observed end-to-end symptoms during failures include both negative symptoms, such as which source-destination pairs are disconnected, as well as positive symptoms, such as which source-destination pairs can still communicate. This information is reported to the network manager by a few selected nodes in the network called reporting nodes; a complete list of symptoms is not required. Using this information, CMC outputs a hypothesis list which consists of a set of network elements whose failures are consistent with the symptoms.

To solve the issue of run-time complexity, CMC adopts a greedy approach when generating the hypothesis list of faulty network elements, as opposed to the combinatorial approach in netCSI. Our greedy approach is similar to a fault diagnosis algorithm called MAX-COVERAGE (MC) [10], which is developed to diagnose black holes or silent failures (independent failures) in IP networks. During clustered failures, the performance of MC degrades significantly-in particular it produces a prohibitively high number of false negatives (see Section V-C1). To overcome this limitation, CMC uses clusters of objects instead of single objects when forming the hypothesis list.

The major contributions of CMC include:

- Clustering models: To diagnose large-scale failures, CMC selects clusters of objects greedily based on clustering models. We propose two different clustering models based on the information available to the network manager (see Section IV-A for further details).

- Utilizing positive symptoms: Unlike MC which uses only negative symptoms, CMC considers both positive and negative symptoms. As a result, CMC outputs a hypothesis list with fewer false positives. In our experiments, we notice that false positives are reduced on average by $45 \%$ with inclusion of positive symptoms.

- Coping with partial symptom information: During 
large-scale failures, it is very unlikely that complete end-to-end symptoms will be available because some of the reporting nodes may not be able to reach the network manager. Unlike the existing fault diagnosis algorithms [2], [3], [10] that assume availability of complete and accurate information at the network manager, CMC specifically addresses the issue of incomplete end-toend symptoms due to a limited number of reporting nodes. ${ }^{1}$ In our simulation results, when compared to MC, only half and $10 \%$ of the reporting nodes are required by $\mathrm{CMC}$ in realistic and random topologies, respectively, to achieve $100 \%$ accuracy.

- Ranking scheme: We develop a ranking scheme as part of CMC to post-process the hypothesis list in the case of large-scale failures. With ranking, CMC results in a better hypothesis list with fewer false positives.

To show the benefits of our algorithm, we compare CMC with two state-of-the-art algorithms (MC and netCSI) through various simulations (see Section V-C). We observe that under clustered failures, CMC outputs a hypothesis list with a significantly lower number of false negatives and a similar or slightly higher number of false positives than MC. In most failure scenarios, we believe that false negatives are far more significant than false positives, because they can go completely undetected or could be very expensive to localize. Therefore, to decrease the number of false negatives during large-scale failures, CMC is willing to pay a small extra price in terms of false positives. Also, when compared to netCSI, the run-time complexity of CMC reduces dramatically to achieve the same level of accuracyfor example, the run-time of CMC is lower than the runtime of netCSI by up to two orders of magnitude when the number of reporting nodes is small (see Section V-C6).

While CMC is better suited for diagnosing large-scale clustered failures, we also observe that MC performs better when localizing independent failures. Therefore, we also propose Adaptive-MAX-COVERAGE (AMC), which employs a Bayesian decision technique as a pre-processing step to classify network failures into independent or clustered based on end-to-end symptoms. AMC employs either MC or CMC depending on the type of failure. Our simulation results show the effectiveness of this adaptive approach over a series of both independent and clustered failures.

The remainder of the paper is organized as follows. We give the background of our work through explaining a few existing algorithms in Section II. We present a system model and an example to illustrate the high-level concepts and novelties of our algorithm in Section III. Then, the three phases of CMC are described in Section IV, and the evaluation of CMC through simulation study is given in $\mathrm{V}$. We also propose an adaptive algorithm called AMC in Section VI, and show the simulation results of AMC in

\footnotetext{
${ }^{1}$ In our experiments, we select reporting nodes randomly.
}

Section VII. Finally, we provide related work in Section VIII and conclude the paper in Section IX.

II. BACKGROUND

In this section, we describe two existing fault diagnosis algorithms, MAX-COVERAGE (MC) and netCSI, which are closely related to our algorithm. We also discuss the limitations of these algorithms under large-scale clustered failures.

MC [10] addresses a problem similar to ours, but is proposed to diagnose black holes or silent failures which are independent. The core algorithm of $\mathrm{MC}$ is based on a greedy approach that considers only a set of negative symptoms called a failure signature. It uses a metric called link coverage (similar to $B C$ in Section III-B), which is defined as the number of negative symptoms that are explained due to the failure of a given object.

MC can be described briefly in three steps. The first step is to iteratively pick an object that has the maximum link coverage. The second step is to remove the symptoms from the failure signature that are explained by this object (picked in first step). The third step is to repeat this process until there are no unexplained negative symptoms in the failure signature. Finally, it outputs a list of objects which is a minimum set of objects that explains all negative symptoms in the failure signature.

MC localizes both single and multiple independent failures. It diagnoses multiple simultaneous failures by dividing the set of a failure signature into different subsets, and each set is explained by one of the multiple failures. However, in large-scale failures, there is a large number of simultaneous failures which are geographically close to each other. In addition, there is high overlapping among negative symptoms that are explained by failure objects. Hence, under clustered failures, the explanation with a minimum set of objects will miss failures, and thus MC may result in a large number of false negatives. As explained in Section I, a high number of false negatives is unacceptable during large-scale failures, since the network manager cannot localize them all or must incur a high cost to diagnose them.

netCSI [13], a combinatorial approach based algorithm, was proposed to diagnose large-scale failures. This is a two step algorithm that generates a hypotheses list which has different possible combinations of objects that could have failed during large-scale failures. In addition, a ranking algoirthm is proposed to localize the massive failures efficiently. netCSI resulted in large gains in accuracy over MC when there are limited number of reporting nodes, but this comes at the cost of run-time. netCSI has a high run-time due to exponential complexity of the combinatorial algorithm. This is undesirable, especially in the case of large networks.

\section{PROBlem AND Motivation}

In this section, we present our system model and network measures used in our algorithm, and describe our approach 
at a high level through an example. Our algorithm diagnoses large-scale failures using the following inputs: the path information (topology) and end-to-end symptoms from reporting nodes.

A. System Model

We consider a network with $n$ nodes, represented by the set $\mathcal{N}$, where nodes are connected to each other by links. There are $l$ links in the network, represented by the set $\mathcal{L}$. We define the term objects to represent either nodes or links in the network. The set of objects is denoted by $O$ which is the union of $\mathcal{N}$ and $\mathcal{L}$. In addition, these objects are divided into clusters based on a specific attribute, where there are $c$ clusters in total, each of which can have a variable number of objects, and are represented by $\mathcal{C}=\left\{C_{1}, C_{2}, C_{3}, \ldots, C_{c}\right\}$. Moreover, these clusters are not mutually exclusive and can overlap with each other.

We assume nodes communicate with each other over multiple routes. The set of source nodes is represented by $S=\left\{s_{1}, s_{2}, s_{3}, \ldots, s_{m}\right\}$, and $S \subseteq \mathcal{N}$. All the possible routes from a source node $s_{i} \in S$ to $k$ different destinations, denoted by $D_{i}=\left\{d_{1}, d_{2}, \ldots, d_{k}\right\}$, are known to the network manager. The routes from source node $s_{i}$ to a destination node $d_{j} \in D_{i}$ are given by $X_{i, j}=\left\{x_{i, j}^{1}, x_{i, j}^{2}, \ldots, x_{i, j}^{e}\right\}$. The $q$-th route is represented by $x_{i, j}^{q}$ and is stored as a set of objects in $O$ that are present along the route. If any of the objects along a route is faulty, then that route is disconnected; otherwise it is considered a connected route. This path information between a source node $s_{i} \in S$ and its corresponding destination nodes is collected periodically, and updates are sent to the network manager.

The end-to-end symptom information represents sources' connectivity and dis-connectivity to their respective destination nodes. A destination node $d_{j} \in D_{i}$ is regarded as connected to $s_{i}$ if there exists at least one connected route in $X_{i, j}$, and disconnected if all the routes in $X_{i, j}$ have at least one failure. Symptoms corresponding to a source node $s_{i} \in S$ can be collected by probing destination nodes that are present in $D_{i}$.

Source nodes that report end-to-end symptoms and the path information to the network manager are called reporting nodes. In practice, the collection of symptom information involves high overhead in terms of network management traffic. In addition, gathering information from all reporting nodes during a massive failure may not be possible. Hence we address the fault diagnosis problem under partial information due to limited reporting nodes in the network, not assuming the availability of the complete end-to-end symptoms from all nodes.

\section{B. Network Measures}

In this paper, we use two important network measures of graph theory: betweenness centrality $(B C)$ and group betweenness centrality $(G B C)$. $B C$ for an object $o_{i} \in O$ is defined as the fraction of paths that pass through $o_{i}$ out of all the paths between different nodes in the network. ${ }^{2}$ In the above definition, the considered paths between nodes are the ones which are available to the network manager from reporting nodes. Moreover, the paths are considered to be loop-free. $G B C$ is a network measure for a cluster of objects, $c_{i} \in \mathcal{C}$, in the network. It is defined as the fraction of paths that pass through $c_{i}$ out of all the paths between different nodes in the network. The mathematical definitions for $B C\left(o_{i}\right)$ and $G B C\left(C_{i}\right)$ are,

$$
\begin{aligned}
B C\left(o_{i}\right) & =\sum_{n_{j} \neq n_{k}} \frac{p_{n_{j}, n_{k}}\left(o_{i}\right)}{p_{n_{j}, n_{k}}} \\
G B C\left(c_{i}\right) & =\sum_{n_{j} \neq n_{k}} \frac{p_{n_{j}, n_{k}}\left(c_{i}\right)}{p_{n_{j}, n_{k}}}
\end{aligned}
$$

where $p_{n_{j}, n_{k}}$ is the total number of the paths between nodes $n_{j}$ and $n_{k}, p_{n_{j}, n_{k}}\left(o_{i}\right)$ is the number of paths between nodes $n_{j}$ and $n_{k}$ that pass through $o_{i}$, and $p_{n_{j}, n_{k}}\left(C_{i}\right)$ is the number of paths between nodes $n_{j}$ and $n_{k}$ that pass through $C_{i} . B C$ is used in CMC (Section IV-B), and $G B C$ is used in AMC while analyzing the failure data (Section VI-B).

\section{Illustration with an Example}

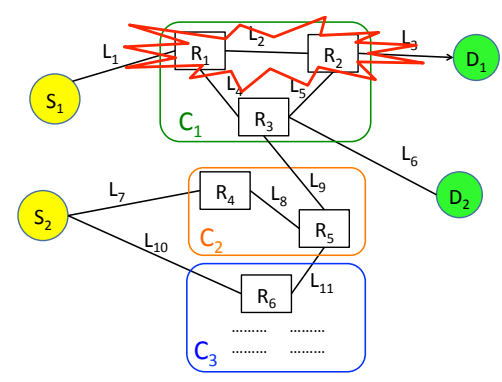

Fig. 1. An example network scenario

Consider the network in Figure 1, in which there are two sources, $S_{1}$ and $S_{2}$, each connected to two destination nodes, $D_{1}$ and $D_{2}$. The routers are divided into 3 clusters: $C_{1}\left(R_{1}, R_{2}, R_{3}\right), C_{2}\left(R_{4}, R_{5}\right)$ and $C_{3}\left(R_{6}, ..\right)$ as shown in Figure 1. The available routes at the network manager for all source-destination pairs are given in Table I. Please note that this is not the list of all possible routes between different source and destination pairs. In this example, we only consider the intermediate routers as objects in the paths. Suppose a large scale failure has occured and routers $R_{1}$ and $R_{2}$ have failed. We observe the following symptoms: source node $S_{1}$ is disconnected from both the destination nodes $D_{1}$ and $D_{2}$, and source node $S_{2}$ is disconnected from destination node $D_{1}$, but is connected to destination node $D_{2}$.

We obtain different hypothesis lists given in Table III through various algorithms: MAX-COVERAGE (MC),

\footnotetext{
${ }^{2} B C$ is originally defined for shortest paths between instead of paths available at the network manager. The same is the case with $G B C$.
} 


\begin{tabular}{|l|l|}
\hline (SRC,DEST) & Routes \\
\hline$\left(S_{1}, D_{1}\right)$ & $r_{S_{1}, D_{1}}^{1}:\left(R_{1}, R_{2}\right)$ \\
& $r_{S_{1}, D_{1}}^{2}:\left(R_{1}, R_{3}, R_{2}\right)$ \\
\hline$\left(S_{1}, D_{2}\right)$ & $r_{S_{1}, D_{2}}^{1}:\left(R_{1}, R_{3}\right)$ \\
& $r_{S_{1}, D_{2}}^{2}:\left(R_{1}, R_{2}, R_{3}\right)$ \\
\hline$\left(S_{2}, D_{1}\right)$ & $r_{S_{2}, D_{1}}^{1}:\left(R_{4}, R_{5}, R_{3}, R_{2}\right)$ \\
& $r_{S_{2}, D_{1}}^{2}:\left(R_{6}, R_{5}, R_{3}, R_{2}\right)$ \\
\hline$\left(S_{2}, D_{2}\right)$ & $r_{S_{2}, D_{2}}^{1}:\left(R_{4}, R_{5}, R_{3}\right)$ \\
& $r_{S_{2}, D_{2}}^{2}:\left(R_{6}, R_{5}, R_{3}\right)$ \\
\hline
\end{tabular}

Table I

AVAILABLE ROUTES BETWEEN

THE NODES

Cluster MAX-COVERAGE (CMC) with only negative symptoms, and with both negative and positive symptoms. Given the negative symptoms, the $B C$ values for various routers are listed in Table II. MC selects the router or routers with maximum $B C$ value, i.e., in our example $R_{2}$ and $R_{3}$ have maximum $B C$ values, and all negative symptoms are explained by both $R_{2}$ and $R_{3}$. Therefore, MC localizes only $R_{2}$, but not $R_{1}$ which has failed. Hence, $R_{1}$ is a false negative.

CMC with only negative symptoms selects the cluster that belong to $R_{2}$ and $R_{3}$, i.e., cluster $C_{1}$ which includes routers $R_{1}, R_{2}$, and $R_{3}$. Hence, the hypothesis list of CMC with only negative symptoms will have no false negatives, but contains $R_{3}$ which is a false positive. Using the information of a positive symptom that says source $S_{2}$ is connected to $D_{2}$ in CMC, we can recognize that router $R_{3}$ is a false positive and eliminate from the hypothesis list since it is present in both the routes between $S_{2}$ and $D_{2}$. This shows that CMC with negative symptoms has fewer false negatives than $\mathrm{MC}$, and the false positives in the hypothesis list can be further reduced using positive symptoms.

\begin{tabular}{|l|l|l|}
\hline $\mathrm{MC}$ & $\begin{array}{l}\mathrm{CMC} \text { with negative } \\
\text { symptoms }\end{array}$ & $\begin{array}{l}\mathrm{CMC} \text { with negative } \\
\text { and positive symptoms }\end{array}$ \\
\hline$\left(R_{2}, R_{3}\right)$ & $\left(R_{1}, R_{2}, R_{3}\right)$ & $\left(R_{1}, R_{2}\right)$ \\
\hline
\end{tabular}

Table III

HYPOTHESIS LISTS FOR VARIOUS ALGORITHMS

\section{Cluster MAX-COVERAGE}

CMC localizes large-scale failures in networks given endto-end observed symptoms and the paths among different nodes that are collected from reporting nodes. There are three phases in CMC. The first phase uses a greedy approach and clustering models to generate a hypothesis list using only negative symptoms. This is called the Hypothesis generation phase. In the second phase, we remove the extra objects (false positives) in the hypothesis list by identifying impossible situations using positive symptoms in the network; this is called the DEC_FP phase. Finally in the third phase, we divide the objects in the resulting hypothesis list into clusters and rank them. We call this the Ranking phase. The pseudo-code of CMC is given in Figure 2. Please look at the italicized text in the pseudo-code to recognize the novelty of CMC algorithm. We use the variables (showed in parentheses) in pseudo-code to explain our algorithm.
We first explain the clustering models that are used in $\mathrm{CMC}$ and then describe the three phases of CMC.

\section{A. Clustering Models}

Clusters of objects are selected using different clustering models in CMC. The network may be divided into different clusters based on various attributes of the network. For example, objects in the same building or an area may be considered to be in one cluster, or objects enclosed in a specified geographical area may be labeled as one cluster. Given the clustered failures, it is most likely that all objects in a cluster may be either faulty or not faulty, collectively.

Alternatively, we can also consider that objects within a certain distance metric from each other, e.g., physical distance or hop count, are most likely to fail together. Hence, we introduce two clustering models based on the information available to the network manager: one with complete information about clusters called the Cluster information model and another with distance information between objects called the Estimated cluster information model.

1) Cluster Information Model: In this model, we assume that all objects in the network are partitioned into clusters and complete information about these clusters is known apriori to the network manager. Our model is generic since the network can be divided into clusters based on any general attribute choosen by the network manager. As explained in Section III-A, we consider that there are $c$ clusters in total and the number of objects in each cluster can vary. Cluster $C_{i}$ with $k$ arbitrary objects is represented by $C_{i}=\left\{o_{1}^{C_{i}}, o_{2}^{C_{i}}, o_{3}^{C_{i}}, \ldots, o_{k}^{C_{i}},\right\}$. In addition, since clusters need not be mutually exclusive, a single object can be present in multiple clusters.

2) Estimated cluster Information Model: Here, we use a metric of distance among objects in the network to estimate clusters. The distance between objects can be measured through various parameters, for example euclidean distance, hop distance, etc. We choose a group of objects as a cluster based on their distance to the reference object which is selected greedily (with maximum $B C$ ). The closeness of the objects is determined using the distance between objects. This model is shown in the following equations:

$$
\begin{array}{r}
D\left(o_{\text {ref }}, o_{j}\right) \leq d_{t h}: o_{j} \in C\left(o_{\text {ref }}\right) \\
\text { otherwise: } o_{j} \notin C\left(o_{\text {ref }}\right)
\end{array}
$$

where $o_{r e f}$ is the reference object that is selected, $o_{j}$ is any object in the set $O, D\left(o_{r e f}, o_{j}\right)$ is the distance between objects $o_{r e f}$ and $o_{j}$, and $d_{t h}$ is the threshold value of the distance.

\section{B. Hypothesis Generation Phase}

The hypothesis generation phase of CMC is inspired from MC, described in Section II. However, CMC is modified to diagnose large-scale failures; the second step of $\mathrm{MC}$ is altered. The pseudo-code for this phase is given in Lines 
2-19 of Figure 2. We pick a cluster of objects rather than a single object, while employing a greedy approach. To be precise, in CMC we choose a cluster of objects that are present around the object with the maximum $B C$ (selObjects in Line 11). This is given in Line 13 of the pseudo-code. In this way, CMC iteratively selects the network objects until all negative symptoms are accounted for. Therefore, in largescale failure scenarios, CMC diagnoses more failed objects when compared to MC.

An alternative straightforward way of selecting clusters of objects is also possible in this phase. Instead of picking an object with the maximum $B C$ value and then selecting clusters, we can directly choose a cluster greedily with maximum $G B C$ value, i.e., a cluster which explains maximum number of negative symptoms. This approach works well when complete cluster information about clusters is available. If only the distance information is available, then there is no reference object for choosing clusters. This results in an infinite number of possible clusters in the network, which makes selection of clusters very difficult. Hence, we use the forementioned approach described in the previous paragraph.

CMC deals with the issue of multiple paths between different nodes in the network, with the help of different topology snapshots (TopologySnapshots) during a given time interval of failure. These snapshots reflect the routing changes that have occured during that interval. This phase is applied for each snapshot to output different hypothesis lists. CMC takes the union of these hypothesis list to output one hypothesis list (unionHypList). MC also tackles the issue of multiple paths in a similar way.

In large-scale failures, CMC results in fewer false negatives compared to MC. However, the hypothesis list may have a high number of false positives, since clusters are chosen instead of single objects. This is more desirable than a high number of false negatives, because in fault management we can probe and confirm false positives, unlike false negatives. Nevertheless, we propose a novel scheme to eliminate false positives from the hypothesis list with the help of observed positive symptoms.

C. DEC FP Phase

In the DEC_FP phase, we decrease the false positives in the hypothesis list. Unlike other works in the area of fault diagnosis [2], [3], an innovative combinatorial scheme with the help of positive symptoms is proposed. This is different from netCSI, which employs a combinatorial approach on various combinations of objects, which are consistent with both positive and negative symptoms. However, the DEC_FP phase takes the list obtained in hypothesis generation phase and employs the combinatorial approach to find combinations, which are not consistent with positive symptoms only. By doing this, CMC diagnoses large-scale failures significantly faster than netCSI. This scheme could also be used for independent failures. We call this the combinatorial

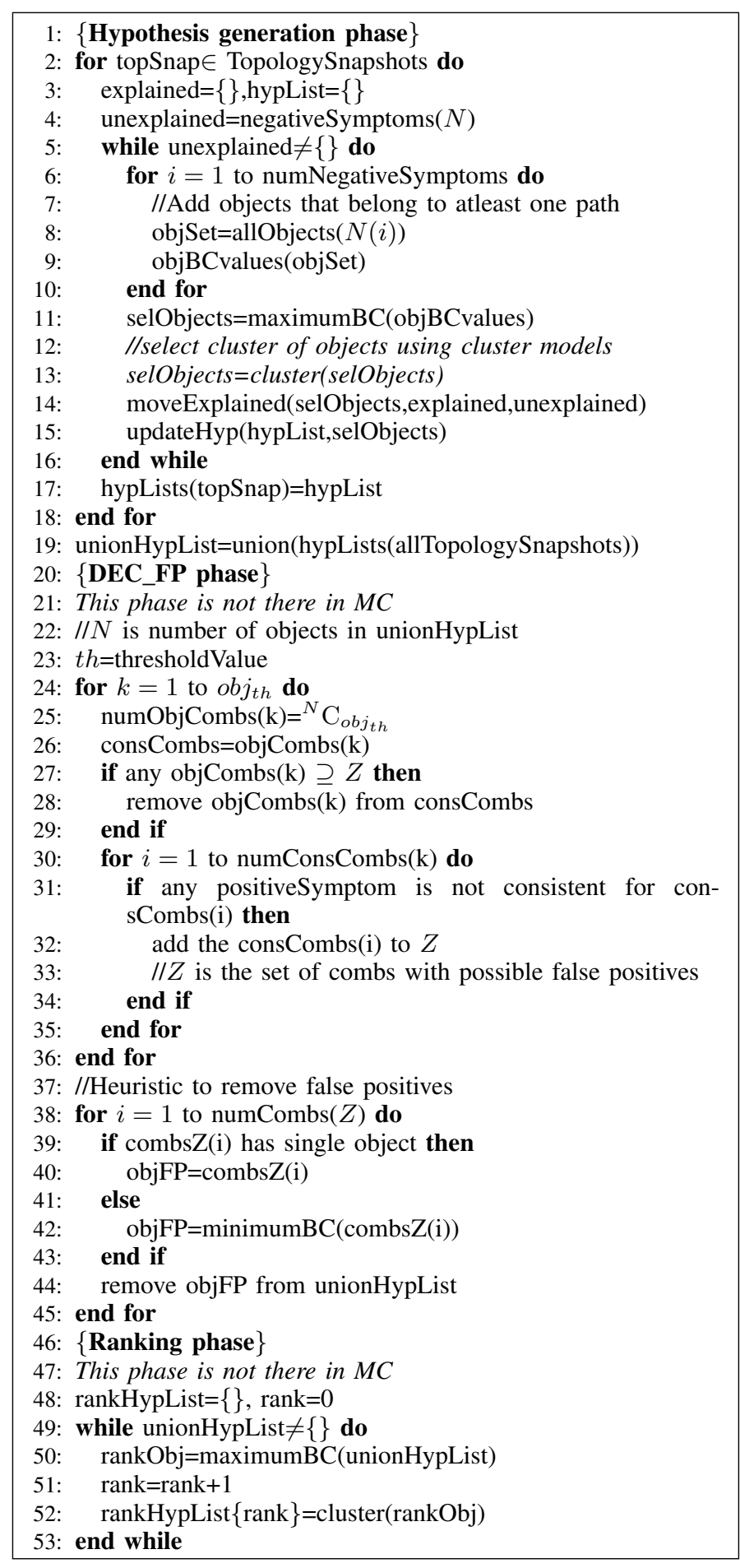

Fig. 2. Pseudo-code of CMC algorithm

algorithm with positive symptoms (COMB_PS). The pseudocode of this phase is given in Lines 21-44 in Figure 2.

1) COMB_PS Algorithm: If there are no false positives in the hypothesis list, then all possible combinations of objects in the hypothesis list (failure combinations) must be consistent for given positive symptoms that are observed by reporting nodes, i.e., the corresponding source-destination 
pairs of positive symptoms should not get disconnected because of failure combinations. In contrast, if there are any false positives in the hypothesis list, some of the combinations could be inconsistent for given positive symptoms. These combinations which make the given positive symptoms infeasible are of interest, because objects of these combinations are likely false positives. The set that constitutes all these combinations is a superset of all false positives present in the hypothesis list. We call this set $Z$ (Line 31 of pseudo-code). Therefore, there are two goals for COMB_PS algorithm. The first one is to find combinations in $Z$ that are not consistent for given positive symptoms, and the second is to recognize false positives in these combinations.

Since the COMB_PS algorithm is a combinatorial approach, the run-time complexity depends on the total number of combinations that are considered. All considered combinations (consCombs) are checked for every positive symptom. If all paths between the corresponding source and destination pair of a positive symptom fail due to objects that are present in the considered combination, then that combination is added to set $Z$. In $Z$, no combination is a superset of another combination, since we are looking for a set that is very close to the set of false positives in the hypothesis list. In other words, the ideal $Z$ is a superset of all false positives and should have a reduced number of objects. Therefore, even though a superset of a combination in $Z$ is inconsistent with the given positive symptoms, we do not have to consider that combination. Hence, we can eliminate the supersets of combinations in $Z$ from the search space, and thereby decrease the run-time complexity of the COMB_PS algorithm. The corresponding code for this elimination is given in Lines 25-28 of Figure 2. In addition, to decrease the run-time of our algorithm we also search only a limited number of combinations, i.e., the combinations with number of objects less than a threshold number called obj_th. In Section V-C, we analyze the effect of $o b j \_t h$ on the run-time of our algorithm.

Combinations in $Z$ include both single-object (only one object) and multiple-object (more than one object) combinations. In single-object combinations, the given object is solely responsible for the inconsistency with positive symptoms. Hence, it is a definite false positive and can be removed from the hypothesis list. However, in multipleobject combinations, we do not know which object or objects have caused the inconsistency in the case of positive symptoms, because we consider multiple paths between nodes. If we eliminate all objects of the combination, we may incur extra false negatives. To avoid this problem, we introduce a heuristic to remove the objects in the hypothesis list that are most probably false positives. Our heuristic is based on the network measure $B C$ (Section III-B). In each combination, we remove the link with the lowest $B C$ value from the hypothesis list. This will decrease the false positives, but cannot completely remove them from the hypothesis list. This heuristic is shown in Lines 36-44 of the pseudo-code. CMC employed with this heuristic approach is called CMC_POS.

\section{Ranking Phase}

We introduce a ranking scheme in this phase for more efficient diagnosis of large-scale failures. The aim of our ranking scheme is to output a hypothesis list with fewer false positives. The ranking scheme is based on $B C$ values of objects and clustering models (Sections III-B,IV-A). Our scheme assigns the highest rank to an unassigned object with the maximum $B C$ value $(r a n k O b j)$ in the hypothesis list, along with the rest of the objects that belong to the same cluster (rankHypList). The clusters are picked according to the given clustering model. We continue this process until all the objects in the hypothesis list are exhausted. Hence, in this phase, we are essentially dividing the objects in the hypothesis list into different clusters, and ranking them. The ranking phase is shown in Lines $46-51$ of the pseudo-code in Figure 2.

Once we have these ranked clusters, we select the clusters which have a lower rank than some threshold rank and group them into a new hypothesis list. This threshold rank is selected by the network manager, and it is called the cumulative rank. The resulting truncated hypothesis list will have fewer false positives when compared to the original hypothesis list. Therefore, false positives are further reduced in this phase. We evaluate our ranking scheme and describe its advantages in Sections V-C3 and V-B.

\section{EVALUATION FOR CMC}

\section{A. Simulation setup}

We evaluate the performance of CMC for two different types of topologies, random and realistic. We run experiments with random topology networks that are generated by placing 100 nodes at random locations in a 2-dimensional space. The realistic topology is taken from Rocketfuel trace data provided by University of Washington [14]. A network with 315 backbone routers that are connected by 972 links in an ISP (Sprint in US) has been considered for our simulations.

In both the networks, multiple paths are generated among all nodes using Dijkstra's algorithm. We generate these paths in multiple instances by failing certain nodes in each instance. The number of multiple paths between any two nodes are less than a threshold number (in our experiments 5 is the threshold). In our experiments without loss of generality, we only consider links as objects in a path, while collecting the path information. In every experiment, a certain number of reporting nodes are selected randomly out of all nodes to report end-to-end symptoms. In addition, we ran 15 trials in each experiment and present the aggregated results over all the trials. 
For simulation purposes, clustering is done in different ways for networks with random and realistic topologies. In random networks, the network is divided into different disjoint clusters using a $k$-means clustering algorithm. With the number of clusters $(k)$ as the input, the $k$-means clustering algorithm generates $k$ clusters, each with a variable number of nodes. For the network with realistic topology, all the nodes in a particular city are regarded as one cluster; there are 24 clusters in total.

We simulate two types of failure modes: random cluster failure and random space failure. In the random cluster failure mode, we choose a random cluster out of all the clusters given in the network and fail all objects in that particular cluster. This failure mode mimics a realistic scenario where all objects in a building (considered as one cluster) may fail due to various reasons. For random space failure mode, a circle with a certain radius $r$ is randomly choosen, and all objects within that circle are failed. This scenario is similar to a case where objects that are geographically adjacent in multiple clusters can fail together, or a group of objects which are geographically close in a large wireless network, can fail simultaneously.

In Section $\mathrm{V}-\mathrm{C}$ we evaluate and compare the following algorithms: $\mathrm{CMC}$ with only the first phase, i.e., with no positive symptoms (CMC_NO_POS), CMC with both first and second phases, i.e., with both positive and negative symptoms (CMC_POS), MC, and netCSI.

\section{B. Performance Metrics}

CMC outputs a hypothesis list that consists of objects which have possibly failed. In this context, we define three metrics which we compare to the ground truth during largescale failures: false negatives, false positives, and accuracy. False negatives (FNs) are defined as the fraction of objects in the ground truth that are not present in the hypothesis list. False positives (FPs) are defined as the fraction of objects in the hypothesis list which are not part of the ground truth. Accuracy is the complement of false negatives, i.e., fraction of objects in the ground truth that are diagnosed correctly. Since we use a ranking scheme in CMC, we define these terms for a hypothesis list with various ranked clusters.

To decrease the false positives, we truncate our hypothesis list so that it contains only a few highly ranked clusters. In this context, we define cumulative rank (CR) as the rank above which all the clusters constitute the truncated hypothesis list at the end of third phase.

\section{Results}

1) Performance of $C M C$ : To evaluate and compare the performance of all algorithms, we simulate random cluster failure mode with the cluster information model. We present results for networks with random and realistic topologies, and with the number of reporting nodes $=20$ and 25 for both topologies, respectively. In the case of random topolgy we consider three instances of $k$ (number of clusters $=40,30,20)$.

As shown in Figure 3(a), CMC_NO_POS results in many fewer false negatives than $\mathrm{MC}$, but incurs more false positives as shown in Figure 3(b). CMC_POS reduces the false positives, while keeping false negatives almost constant. This shows the significance of including positive symptoms in failure diagnosis. We observe similar trends in the network with realistic topolgy, but with high gains as shown in Figures 7(a) and 7(b). We now evaluate CMC_POS while varying the parameter $o b j_{t h}$.

2) Varying $o b j_{t h}$ in DEC_FP Phase: We vary the parameter $o b j_{t h}$ (explained in Section IV-C1) and evaluate the performance of CMC under random cluster failure mode with the cluster information model. In the network, the number of reporting nodes $=20$ and the number of clusters $=20$. In Figure 5, with the increase in the value of $o b j_{t h}$, FPs decrease slightly, and there is a minute increase in FNs. However, the run-time of CMC increases exponentially with the increase in $o b j_{t h}$. Therefore, truncating the number of considered combinations with the help of $o b j_{t h}$ decreases the run-time of CMC with minimal effect on it's performance. Hence, in the rest of the paper, we use a value of $o b j_{t h}=3$ for CMC_POS.

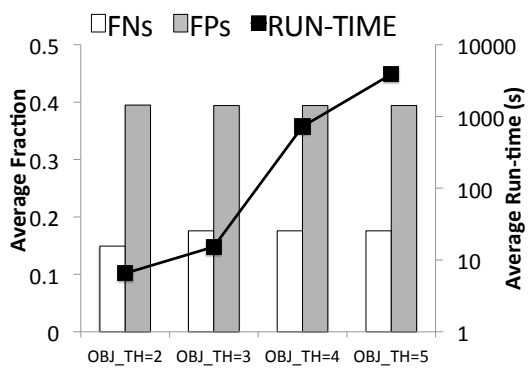

Fig. 5. Performance of CMC while varying $o b j_{t h}$

3) Ranking Phase: Here, we discuss the benefits of the ranking phase. This can be employed with both CMC_NO_POS and CMC_POS. We show results for random topology with 20 clusters in Figure 6. We compare the following cases: no_ranking (i.e., Ranking is not employed), $C R=1, C R=2$, and $C R=3$. Both CMC_NO_POS and CMC_POS, with $C R=1$ have the same FNs as no_ranking, but FPs are lower than the case no_ranking. This result shows that the ranking scheme can diagnose the same number of failed objects as without the ranking scheme, but with fewer false postives. In this particular scenario, we observe the case $C R=1$, but in general, $C R$ with a very low value can achieve this result.

4) Clustering Models: In Figure 3, we show the results for random cluster failure mode with both cluster information and estimated cluster information models. Similarly, in Figure 4, the results are shown for only random space failure mode. 20 reporting nodes are selected in all cases. In both figures, we observe that when the clustering model and 


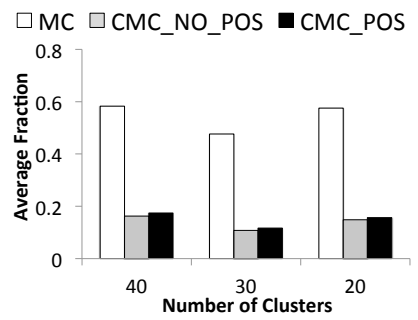

(a) False Negatives

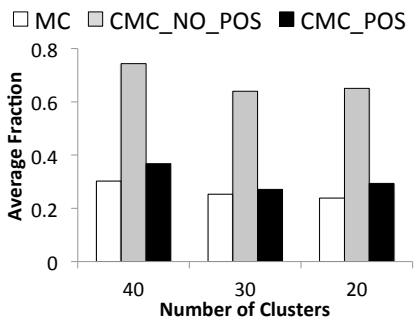

(b) False Positives

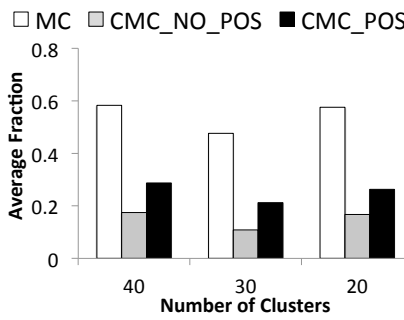

(c) False Negatives

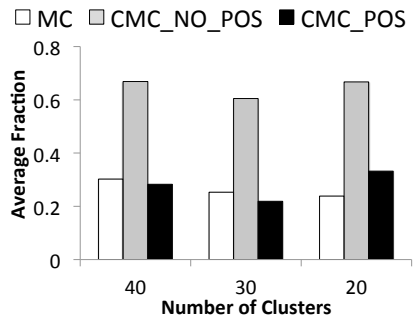

(d) False Positives

Fig. 3. Random cluster failures in random networks: (a) \& (b) are with complete cluster information; (c) \& (d) are with object distance information

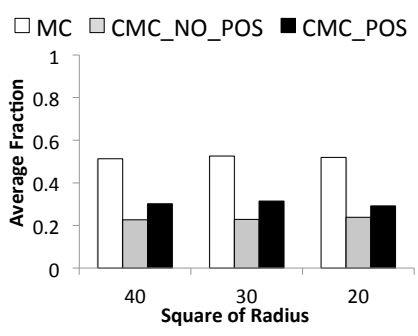

(a) False Negatives

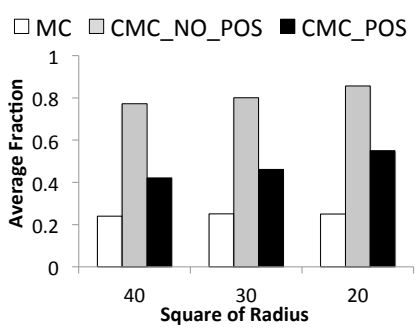

(b) False Positives

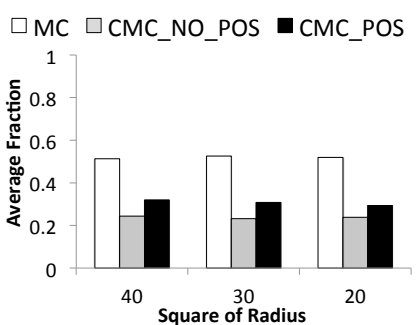

(c) False Negatives

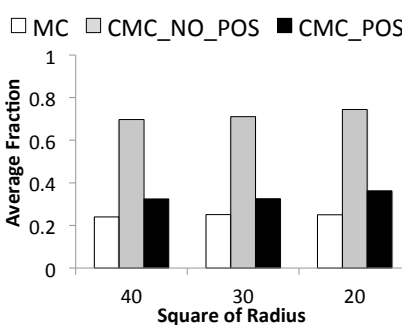

(d) False Positives

Fig. 4. Random space failures in random networks: (a) \& (b) are with complete cluster information; (c) \& (d) are with object distance information

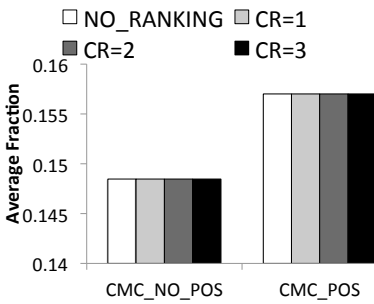

(a) False Negatives

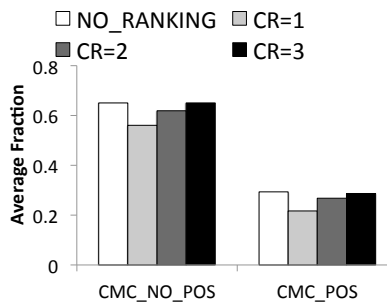

(b) False Positives
Fig. 6. Performance of Ranking Scheme in CMC

failure mode are consistent with each other, the performance of CMC is much better than the case when they are not. Furthermore, in the case of discrepancy in the failure mode and cluster information model, false negatives are not affected at all when compared to the discrepancy with estimated cluster information model. Since false negatives may go undetected by the network manager in contrast to false positives which can be detected with the help of probing, we claim that having the cluster information model is better than having only the estimated clustering model. The results for network with realistic topology in Figure 7 show that they follow the same trend as in networks with random topology.

5) Varying Reporting Nodes: Now, we focus on the performance of various algorithms while varying the number of reporting nodes. The accuracy of CMC_POS, CMC_NO_POS, MC, and netCSI are evaluated for both types of networks, as shown in Figure 8. The accuracy of fault diagnosis increases with an increase in the number of reporting nodes. In a random topology of 100 nodes as shown in Figure 8(a), we observe that CMC_NO_POS and CMC_POS accomplish $100 \%$ accuracy with 40 reporting

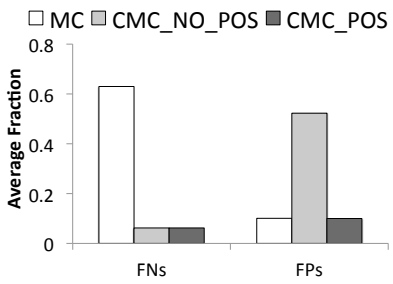

(a) Complete cluster information

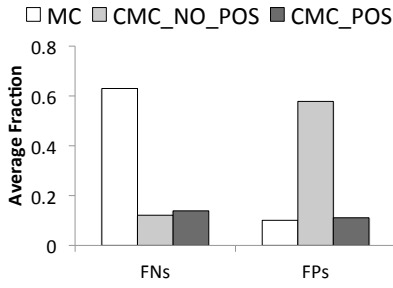

(b) Object Distance Information
Fig. 7. Random cluster failures in network with realistic topology

nodes (approximately), whereas MC requires 70 reporting nodes to diagnose all objects in the ground truth. In a network of 315 nodes with a realistic topology, we observe high gains, i.e., with 25 reporting nodes, CMC_NO_POS and CMC_POS achieve an accuracy of almost $100 \%$. However, in the case of MC, it requires 275 reporting nodes to accomplish the same level of accuracy. In both topologies, a high number of reporting nodes results in a higher burden on the network manager while collecting symptoms and paths, in terms of network overhead and probing costs. Therefore, both CMC_NO_POS and CMC_POS achieve $100 \%$ accuracy with very low overhead when compared to MC. Please note that in Figures 8(a) and 8(b), the $\mathrm{X}$-axis is different, because Figure 8(a) is for random topology which has 100 nodes in the network and Figure 8(b) represents realistic topology that consists of 315 nodes in the network.

netCSI achieves almost the same accuracy as both CMC_NO_POS and CMC_POS, but incurs a high runtime, since it employs a combinatorial approach instead of a greedy approach. This will be explained in the next section with experimental results. 


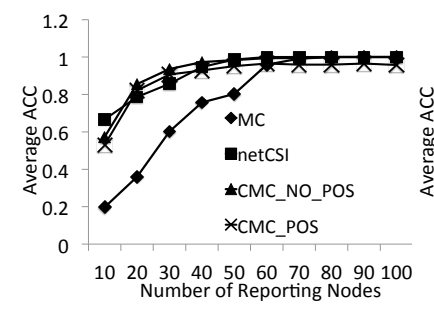

(a) Random topology

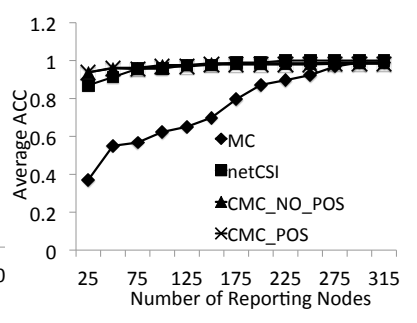

(b) Realistic topology
Fig. 8. Accuracy vs number of reporting nodes

6) Run-time: We show the plots for run-time of different algorithms in Figure 9 for both random and realistic topologies. In Figure 9(a), netCSI incurs the maximum run-time of all the algorithms, since it is a combinatorial approach. CMC_POS has higher run-time than CMC_NO_POS and MC, because of the DEC_FP phase. Even though CMC_POS involves a combinatorial approach, it is better than netCSI in terms of run-time because the number of considered combinations required in CMC_POS are far less than netCSI, i.e., the $o b j_{t h}$ value is very low in the case of CMC_POS. MC has the lowest run-time of all, but its performance deteriorates under large-scale failures as explained in Section $\mathrm{V}-\mathrm{C} 1$. For a network with a realistic topology, as shown in Figure 9(b), we observe similar trends, but the run-time is very high because the number of objects in the network is high when compared to random topology.

Therefore, if the performance of network management does not suffer from high false positives, we can apply only CMC_NO_POS, which results in low FNs compared to $\mathrm{MC}$, but has almost same run-time as MC. Otherwise, if the network manager wants to diagnose large-scale failures with low false positives, i.e., by applying CMC_POS, then extra run-time is incurred.

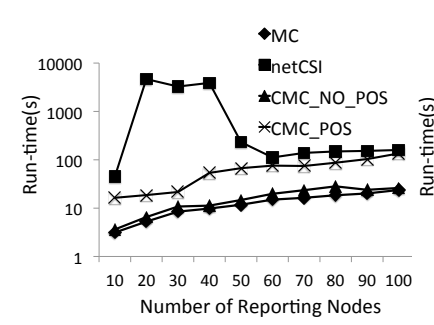

(a) Random topology

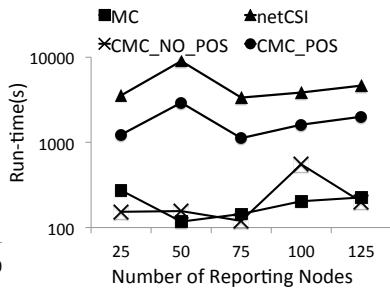

(b) Realistic topology
Fig. 9. Run-time comparison of all algorithms

\section{AdAPTIVE-MAX-COVERAGE}

As our results in Section V-C have shown, CMC outperforms MC for clustered failures. However, in the case of independent failures, MC outperforms CMC in terms of false positives, as shown in Table IV (Fraction of FPs and FNs). These results are produced using the experimental setup given in Section V-A. For clustered failures, we simulated random cluster failure mode (Section V-A), and

\begin{tabular}{|l|l|l|}
\hline & Independent & Clustered \\
\hline MC & $\mathrm{FP}=0.22$ & $\mathrm{FP}=0.3$ \\
& $\mathrm{FN}=0.2$ & $\mathrm{FN}=0.59$ \\
\hline CMC-POS & $\mathrm{FP}=0.85$ & $\mathrm{FP}=0.33$ \\
& $\mathrm{FN}=0.19$ & $\mathrm{FN}=0.18$ \\
\hline \multicolumn{3}{|c|}{ Table IV }
\end{tabular}

PERFORMANCE OF MC AND CMC VS DIFFERENT FAILURES

for independent failures, we failed links that are selected randomly.

Therefore, to support case in which there are both independent and clustered failures, we propose a hybrid fault diagnosis approach called Adaptive MAX-COVERAGE (AMC). The key element of AMC is determining whether a failure is independent or clustered, so that an appropriate fault diagnosis algorithm can be employed accordingly. AMC classifies failure types with the help of the Bayes decision technique. This technique makes use of end-toend negative symptoms in the network during failures to differentiate various types of failures.

\section{A. Basic Approach}

Our decision technique uses the classic Bayesian decision theory to classify different failure types. In this paper, we consider two classes of failures: independent and clustered failures. We define two events $I F$ and $C F$, for independent and clustered failures, respectively. In addition, we define a random variable, $X$, to represent the number of negative symptoms in the network due to a failure. Given the probability density functions of $X$ for both independent and clustered failures (prior to the current failure), and the observed negative symptoms due to a current failure in realtime $(n s)$, we use Bayes rule to make the decision about the failure. The following equations explain our technique,

$$
P(I F \mid X=n s)=\frac{P(X=n s \mid I F) P(I F)}{P(X=n s \mid I F) P(I F)+P(X=n s \mid C F) P(C F)}
$$

$$
\begin{gathered}
P(C F \mid X=n s)=\frac{P(X=n s \mid C F) P(C F)}{P(X=n s \mid I F) P(I F)+P(X=n s \mid C F) P(C F)} \\
P(C F \mid X=n s) \geq P(I F \mid X=n s): \text { Clustered failure } \\
P(C F \mid X=n s)<P(I F \mid X=n s): \text { Independent failure }
\end{gathered}
$$

where $P(I F)$ and $P(C F)$ are the prior probabilities of independent and clustered failure events, given by

$$
\begin{gathered}
P(I F)=\frac{\text { Number of Independent failures }}{\text { Total number of failures }} \\
P(C F)=\frac{\text { Number of Clustered failures }}{\text { Total number of failures }} .
\end{gathered}
$$

The prior probabilities, $P(I F)$ and $P(C F)$, for a given period of time are updated after each failure with the help of feedback information, i.e., whether the failure is independent or clustered. The values of $P(X=n s \mid I F)$ and $P(X=n s \mid C F)$ are determined using the probability density functions of $X$ given independent and clustered failure 
events, i.e., $X \mid I F$ and $X \mid C F$. We explain our methodology to evaluate these probability density functions using a database in Section VI-B. Finally, we use the the posterior probabilities, $P(C F \mid X=n s)$ and $P(I F \mid X=n s)$, which are determined with the help of Bayes rule (Equations (3) and (4)), to classify failures as shown in Equation (5).

\section{B. Failure Data}

We utilize a database of failure data that consists of both offline and online data about independent and clustered failures. The database contains the number of end-to-end negative symptoms in the network for both types of failures. This helps us to estimate the probability density functions of random variables, $X \mid I F$ and $X \mid C F$. Offline data are collected from samples of both independent and clustered failures, chosen randomly in the network. The online data are obtained from the feedback given to the network manager when the failures occur in real-time.

For offline data, we use the network measures $B C$ and $G B C$, to determine the end-to-end negative symptoms, instead of enumerating the symptoms by simulating failures. In single object failure case, the number of negative symptoms is equal to $B C$ of the failed object. When multiple objects fail simultaneously and independently, the total number of end-to-end negative symptoms is estimated as the sum of the $B C$ values of the failed objects. For clustered failures, we choose samples that include both random cluster and random space (Section V-A) depending on the available cluster information with the network manager. For both modes, the number of end-to-end negative symptoms is equal to cluster's $G B C$ value. Here, note that to determine the negative symptoms, we are not simulating failures offline which involves high complexity, but instead we are using relevant graph measures.

This offline data enable us to construct the histograms that represent the frequency of number of negative symptoms corresponding to both independent and clustered failures. These histograms are updated with online data after each failure, i.e., using the number of negative symptoms observed in the network whenever a failure occurs in realtime. We use a well-known technique called kernal density estimation [15] to estimate the probability density function of $X \mid I F$ and $X \mid C F$ from histograms.

\section{Cost Function}

To enable efficient and robust fault diagnosis, our Bayes decision technique uses a cost function. The cost function represents the penalities incurred when MC and CMC are applied for different failure types, i.e., false positives and false negatives. A sample cost function is shown in Equation (7a). It is defined for MC and CMC under both independent and clustered failures.

$$
\begin{gathered}
c f=\alpha \times F P+(1-\alpha) \times F N \\
D_{i}=c f_{M C}^{i} P(I F \mid X=n s)+c f_{C M C}^{i} P(C F \mid X=n s)
\end{gathered}
$$

$$
\begin{gathered}
D_{c}=c f_{M C}^{c} P(I F \mid X=n s)+c f_{C M C}^{c} P(C F \mid X=n s) \\
D_{c} \leq D_{i}: \text { Clustered failure } \\
D_{c}>D_{i}: \text { Independent failure }
\end{gathered}
$$

where $\alpha$ in Equation (7a) is a generic parameter that represents the significance of false positives (FPs) and false negatives (FNs) to the network manager (Here we use the fractions of FP and FN in the cost function, rather than the numbers of FPs and FPs). The network manager chooses the appropriate value for $\alpha$ depending on the circumstances in the network. For example, while in most networks FNs are more expensive than FPs since they go undetected through automated network management, in some networks the probing requires manual inspection and hence FPs are more difficult to recover than FNs.

We calculate the costs incurred $D_{i}$ and $D_{c}$ (Equations (7b) and (7c)) for both independent and clustered failures, and accordingly we classify failures as shown in Equations (7d) and (7e). Note that all cost functions $\left(c f_{M C}^{i}, c f_{M C}^{c}\right.$, $c f_{C M C}^{i}$ and $c f_{C M C}^{c}$ ) are defined in terms of the fraction of FPs and FNs, which makes the technique independent of the scale of failures. The values of these cost functions are determined through training data. In our evaluation, the training data consists of random samples of failures that include independent and clustered failures, and both MC and CMC are employed for every sample. In each case the averages of FPs and FNs are used to compute the corresponding cost function value. In addition, we update the values of cost functions with online failure data, i.e., the resulting FPs and FNs whenever failures occur in real-time.

\section{Evaluation of AMC}

We use the same experimental setup given in Section V-A to evaluate AMC. Here, we simulate a series of failures that include both types of failures, i.e., independent and clustered ones. We evaluate AMC with the following parameters: number of reporting nodes $(R)$, and the probability of independent failures $(P)$. The number of reporting nodes signifies the extent of partial information at the network manager, whereas probability of independent failures specifies how often independent failures occur in a series of failures. We use the fraction of false positives and false negatives (FPs and FNs) that are defined in Section V-B as metrics.

We evaluate and compare various fault diagnosis algorithms for three cases. The values of variables for different cases are given in Table V. In CASE-1, every variable has a base value. In next two cases, we vary only one variable in each case as shown in Table V. We do this to understand how different variables affect various algorithms individually. We have given results for a 100 node network with random topology.

Figure 10 shows that $\mathrm{AMC}$ and $\mathrm{CMC}$ algorithms produce fewer FNs than MC in all cases. This is because of the 


\begin{tabular}{|l|l|l|}
\hline CASE-1 & CASE- 2 & CASE-3 \\
\hline$R=20$ & $R=50$ & $R=20$ \\
$P=0.8$ & $P=0.8$ & $P=0.2$ \\
\hline \multicolumn{3}{|c}{ Table V } \\
F VARIABLES IN DIFFERENT CASES
\end{tabular}

large-scale clustered failures that are present in the simulated series of failures. However, FPs in the case of CMC are high when compared to other algorithms as shown in Figure 11 , due to the degradation of its performance during independent failures. The AMC algorithms diagnose the failures efficiently, i.e., resulting in a good number for both FPs and FNs.

In CASE-2, the number of reporting nodes are high, i.e., there is more information at the network manager. Both FPs and FNs decrease as shown in Figures 11 and 10 for MC and AMC algorithms. For CMC, the number of FPs remains high because of performance degradation during independent failures. In CASE-3, unlike CASE-1, clustered failures occur more often than independent failures. We observe that FNs increase for MC, and FPs decrease for CMC, when compared to CASE-1.

In our experiments, we vary the maximum number of independent failures, i.e., maximum number of objects that can fail at a time during independent failures. We observe that the FPs and FNs do not change much for all algorithms, especially for AMC algorithms, i.e., the classification success rates (success rate of our Bayes decision technique) do not change considerably with an increase in the maximum number of independent failures. Furthermore, when we change the connectivity density of the network and hence when the size of clustered failures is increased, FPs and FNs do not vary much for different algorithms, except for MC. This is because MC treats all clustered failures as independent failures.

Introduction of a cost function gives the network manager more control over relative emphasis on FPs and FNs in failure diagnosis. This is shown in the plot in Figure 12 where AMC with a cost function is evaluated for different values of $\alpha$. We consider the parameters of CASE- 1 for this plot. As we increase $\alpha$, FPs decrease, since the penality incurred by FPs have more weight. Similarly, with an increase in $\alpha$ value, the weight for FNs decreases, and therefore FNs increase as shown in Figure 12. In addition, we also show the FPs and FNs in the case of AMC with no cost function in Figure 12; this can also be seen for CASE-1 in Figures 11 and 10.

Here, we can broadly divide the values of $\alpha$ into three different regions as shown in Figure 12. First region is where AMC considers almost every failure as independent, and employs MC. In the second region, AMC will have different classification success rates for various values of $\alpha$, and thus FPs and FNs change accordingly. Finally, in the third region, AMC considers every failure as clustered, and employs CMC.

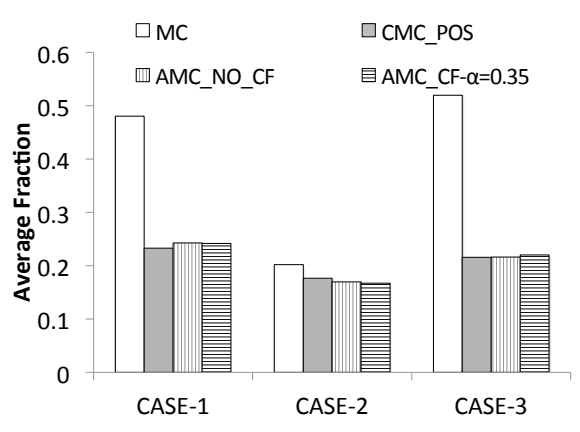

Fig. 10. Evaluation of AMC: False Negatives

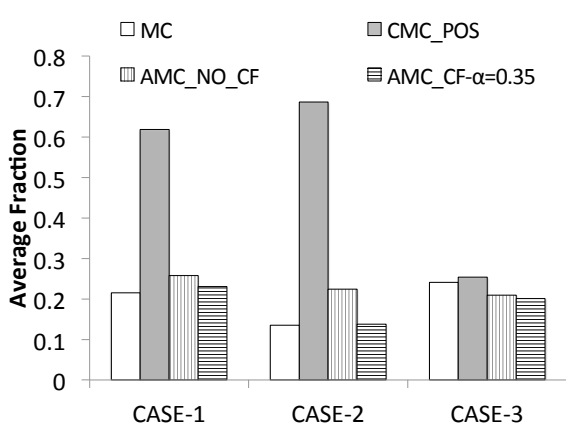

Fig. 11. Evaluation of AMC: False Positives

\section{RELATED WORK}

There is much prior work [1], [2], [10], [16] that address the localization of independent failures of individual components in the network, but there is little focus on large-scale failures. However, there is considerable interest on other various aspects of large-scale failures in current literature [11], [12]. Recently, netCSI [13], a combinatorial based algorithm is proposed to diagnose large-scale failures. However, there is a limitation of run-time in large networks. We propose Cluster MAX-COVERAGE (CMC), to diagnose massive failures accurately under challenges posed by them and solve the issue of run-time.

MAX-COVERAGE (MC) [10], a fault diagnosis algorithm based on SCORE (space correlation engine) [2], focuses on MPLS-over-IP backbone networks. Localization agents use the end-to-end connectivity measurements as alarms and employ spatial correlation techniques to isolate the failures. Since these algorithms are proposed to diagnose independent failures, their performance degrades for large scale failures when there are incomplete symptoms. CMC addresses the problem of incomplete symptoms under largescale failures. Furthermore, CMC uses both negative and positive symptoms, unlike MC and SCORE, which consider only negative symptoms.

Sherlock [16], Shrink [3], Steinder et al [17], and netCSI [13] propose fault diagnosis techniques that use combinatorial algorithms with exponential computational complexity. They propose various optimizations to minimize the complexity of the algorithm. However, in large networks the 


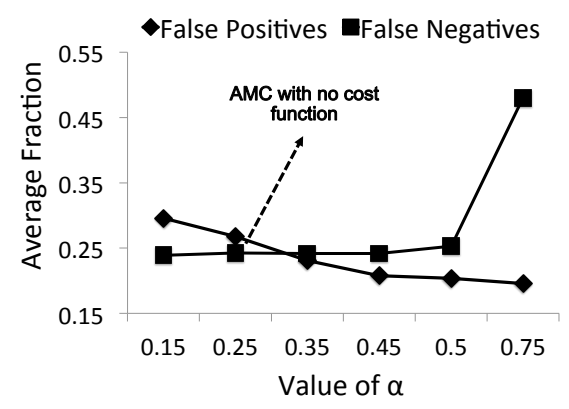

Fig. 12. Evaluation of $\mathrm{AMC}$ w/ cost function while varying $\alpha$

complexity becomes very high. Our algorithm, CMC, is based on a greedy approach, and thus has lower computational complexity than combinatorial approaches. Furthermore, both Sherlock and Shrink assume the availability of complete symptoms, whereas CMC performs well even in cases with incomplete symptoms.

Moreover, in this paper, we recognize the requirement of different fault diagnosis algorithms under both independent and clustered failures, and propose Adaptive MAXCOVERAGE (AMC) algorithm. To the best of our knowledge, AMC is the first algorithm to focus on diagnosing both independent and clustered failures when there is partial symptom information in the network.

\section{Conclusion}

In this paper, we introduced CMC, an algorithm to diagnose faults due to large scale failures accurately and quickly, in spite of having incomplete symptom information. CMC accomplishes $100 \%$ accuracy with one-tenth of the reporting nodes in a random topology and half of the reporting nodes in realistic topology, when compared to MC. This gain is achieved much faster than netCSI-sometimes by two orders of magnitude. CMC utilizes positive symptoms to output a hypotheses list that has $45 \%$ fewer false positives when compared to the case without any positive symptoms. We also propse an adaptive algorithm called AMC to diagnose both independent and clustered failures effectively with the help of Bayesian decision technique. AMC produces a hypothesis list with a low number of false positives and false negatives when compared to $\mathrm{CMC}$ and $\mathrm{MC}$ over a series of failures.

\section{Acknowledgements}

We acknowledge the inputs of Daniel Kifer. This research was sponsored by the U.S. Army Research Laboratory and the U.K. Ministry of Defence and was accomplished under Agreement Number W911NF-06-3-0001. The views and conclusions contained in this document are those of the author(s) and should not be interpreted as representing the official policies, either expressed or implied, of the U.S. Army Research Laboratory, the U.S. Government, the U.K. Ministry of Defence or the U.K. Government. The U.S. and U.K. Governments are authorized to reproduce and distribute reprints for Government purposes notwithstanding any copyright notation hereon. This work was supported in part by the Defense Threat Reduction Agency under grant HDTRA1-10$1-0085$.

\section{REFERENCES}

[1] M. Steinder and A. S. Sethi, "A survey of fault localization techniques in computer networks," Science of Computer Programming, vol. 53, no. 2, pp. 165 - 194, 2004.

[2] R. R. Kompella, J. Yates, A. Greenberg, and A. C. Snoeren, "Ip fault localization via risk modeling," in Networked Systems Design and Implementation, Berkeley, CA, USA, 2005, pp. 57-70.

[3] S. Kandula and D. Katabi, "Shrink: A tool for failure diagnosis in ip networks," in MineNet: ACM SIGCOMM workshop on Mining network data, 2005.

[4] J. Cowie, A. Popescu, and T. Underwood, "Impact of Hurricane Katrina on Internet infrastructure," http://www.renesys.com/tech/presentations/pdf/ Renesys-Katrina-Report-9sep2005.pdf, 2005.

[5] "Hurricane Irene Internet Outages," http://www.huffingtonpost.com/2011/08/27/ hurricane-irene-power-outages_n_939143.html, 2011.

[6] O. Malik, "In Japan, many undersea cables are damaged." 'http://gigaom.com/broadband/ in-japan-many-under-sea-cables-are-damaged/, 2011.

[7] S. LaPerriere, "Taiwan Earthquake Fiber Cuts: A service provider view," http://www.nanog.org/meetings/nanog39/ presentations/laperriere.pdf, 2007.

[8] A. Ogielski and J. Cowie, "Internet routing behavior on $9 / 11$ and in the following weeks," http://www.renesys.com/tech/ presentations/pdf/renesys-030502-NRC-911.pdf, 2002.

[9] C.Wilson, "High altitude electromagnetic pulse(hemp) and high power microwave (hpm) devices: threat assessments," http://www.fas.org/man/crs/RL32544.pdf, 2004.

[10] R. R. Kompella, J. Yates, A. Greenberg, and A. C. Snoeren, "Detection and localization of network black holes," in IEEE Infocom, 2007.

[11] A. Sahoo, K. Kant, and P. Mohapatra, "Improving BGP Convergence Delay for Large-Scale Failures," in Dependable Systems and Networks. Los Alamitos, CA, USA: IEEE Computer Society, 2006, pp. 323-332.

[12] B. Bassiri and S. S. Heydari, "Network survivability in largescale regional failure scenarios," in C3S2E, New York, NY, USA, 2009, pp. 83-87.

[13] S. Tati, S. Rager, B. J. Ko, G. Cao, A. Swami, and T. La Porta, "netCSI: A generic fault diagnosis algorithm for largescale failures in computer networks," in IEEE Symposium on Reliable Distributed Systems, Madrid, Spain, October 2011.

[14] "Rocketfuel Project: Internet topologies," http://www.cs. washington.edu/research/networking/rocketfuel/.

[15] B. W. Silverman, Density estimation for statistics and data analysis. CRC Press, 1986.

[16] P. Bahl, R. Ch, A. Greenberg, S. K, D. A. Maltz, and M. Zhang, "Towards highly reliable enterprise network services via inference of multi-level dependencies," in In SIGCOMM, 2007, pp. 13-24.

[17] M. Steinder and A. S. Sethi, "Probabilistic fault diagnosis in communication systems through incremental hypothesis updating," Comput. Netw., vol. 45, no. 4, pp. 537-562, 2004. 